\title{
A Study of the Spatial Heterogeneity of
}

\section{Some Chemical Properties of Terabhor Back Using GIS}

\author{
Wurood Amer Abdul Amir \\ Amal Radi Jubair \\ Al-Qasim Green University, College of Agriculture \\ wurood1230@gmail.com
}

\author{
ARTICLE INFO \\ Submission date: $13 / 8 / 2018$ \\ Acceptance date :23/9/2018 \\ Publication date: 10/3/2019
}

\begin{abstract}
Done studying the variability of the soils And the heterogeneity of the characteristics of the soils of the Euphrates, where 6 boreons were described and described the horizons of the pidoons as morphological and fundamental, as well as obtaining random samples on four equal depths $25-0 \mathrm{~cm}$, $50-25 \mathrm{~cm}, 75-50 \mathrm{~cm}$ and $100-75 \mathrm{~cm}$. The results of spatial heterogeneity Turbidity and Spatial Distribution Maps The heterogeneity of all the characteristics of chemical faecal soil is indicated by the results. The results indicate the variance of the horizons in the moving distance that describe the heterogeneity of the chemical properties, Electrical conductivity of EC Ranging between 0.48-5.46 and gypsum Ranging between $0.15-4.61$ qualities more heterogeneous, and then followed by organic matter Ranging between 0.27-4.50 and CEC Ranging between 18.66-38.42 .

The least heterogeneous properties were soil $\mathrm{pH}$ Ranging between 7.05- 7.62, followed by calcium carbonate Ranging between 284.28 - 438.71, where the range was high

The results showed that the appropriate model describing the heterogeneity of the chemical properties was the circular model, the appropriate model for describing its variation by $54 \%$, followed by the spherical model of $43 \%$ and the gussin model of $4 \%$.

The soil of the study according to the modern American system 2014 is classified into two levels: Entisols and Mollisols .
\end{abstract}

Keywords: GIS, geological statistics, spatial variations, chemical properties. 


\title{
دراسة التخاير المكانيخ لبهخ الصفات الكيميائية لترب هور عودة بأستخدام نظم المهلومات الجغرافية GIS
}

\author{
أمل راضي جبير \\ ورود عامر عبد الامير \\ كلية الزراعة/جامعة القاسم الخضراء \\ كلية الزراعة/جامعة القاسم الخضراء \\ wurood1230@gmail.com
}

\section{الخلاصة}

تمت دراسة تغاير ترب الهور وتغاير صفات ترب الهور في محافظة ميسان , إذ تم حفر ستة بيدونات ووصفت أفاق البيدونات وصفآ مورفولوجياً أصولياً, وكذللك أستحصال عينات بينية عشوائية على أربعة أعماق متساوية 0-25 سم و 25-50 سم

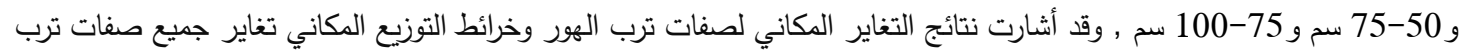
الهور الكيميائية ,فقد اشارت النتائج الى تغاير الافاق في المسافة المؤثرة التي تصف تغاير الصفات الكات الكيميائية , إذ كانت الايصالية الكهربائية EC تتزاوح بين 0.48 - 5.46 والجبس تراوح بين 0.15 - 4.61 أكثر الصفات تغايرآ , ثم تلتها المادة العضوية الذي الذيات

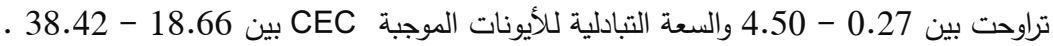

أما الصفات الاقل تغايرآ فقد كان تفاعل التربة pH الذي تراوحت بين 7.05 - 7.62 يليه كاربونات الكالسيوم 284.28 - 28. 438.71 , إذ كانت المسافة المؤثرة Range عالية في تلك الصفات .

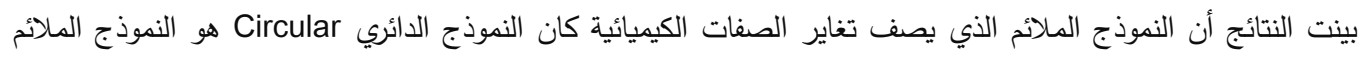

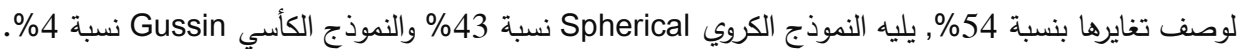
صنفت ترب الدراسة بحسب النظام الأمريكي الحديث 2014 الى رتبتين هما رتبة Entisols ورتبة Mollisols . الكلمات الدالة :GIS, الاحصاء الجيولوجي , التغايرات المكانية , الصفات الكيميائية

\section{المقدمة}

إن مفاهيم دراسات التغاير المكاني في علوم التربة والمستندة على أسس الاحصاء الجيولوجي

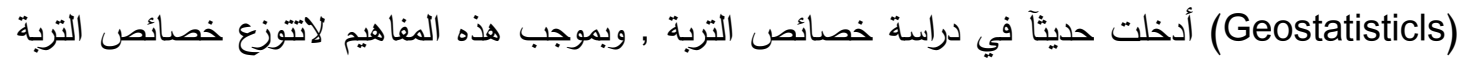

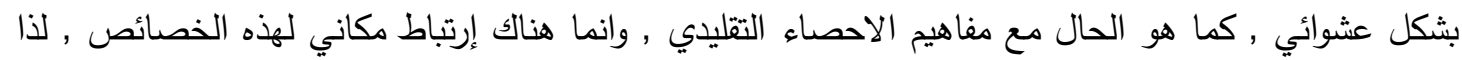
فأن دراسة كل خاصية من خصائص كل تربة تتطلب معرفة الارتباط المكاني ومدى تأثير كل خاصية , وعليه

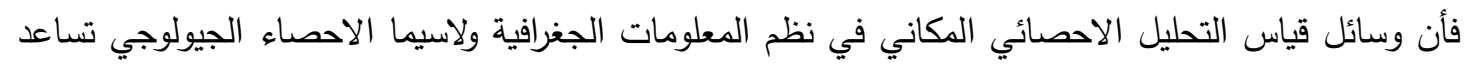
وبكل الوسائل الاحصائية التقليدية [1] • إن إستخدام الاحصاء الجيولوجي عن طريق برنامج GIS يتيح لنا إمكانية إستخدام طرائق Kriging للتنبؤ لإئ

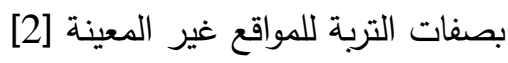
ولأهمية ترب الاهوار تعدً من الأراضي المنخفضة التي تغطيها المياه سواءًٌ في جميع ايام السنة او في بعضها , ولا يوجد فرق واضح بين الاهوار و المستتقعات [3]. إذ يعد هور عودة سابقآ حلقة الوصل بين الاهوار الثمالية الغربية والاهوار الوسطى المركزية عن طريق وليق

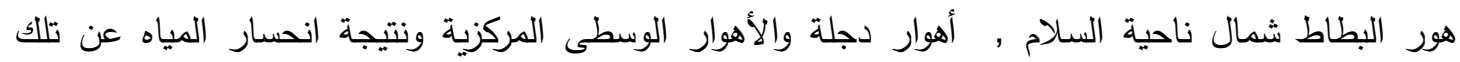
المنطقة لاسباب طبيعية واصطناعية بقي الماء في المناطق المنخفضة كونت أهوار مستقلة سميت بأسماء المناطق والاشخاص من ضمنها هور عودة والسعدية والخراب وأم البقر والصحين والوادية والبطاط وغيرها. 
وأوضح [4] أن التغاير المكاني للتربة ينشأ من عوامل مختلفة أهمها عوامل تكوين التربة وممارسات إدارة التربة ورطوبة التربة .

وبين [5] عند دراسته لترب سيريلانكا ان هناك تغايرآ كبيرآ في درجة تفاعل التربة pH والمادة العضوية.

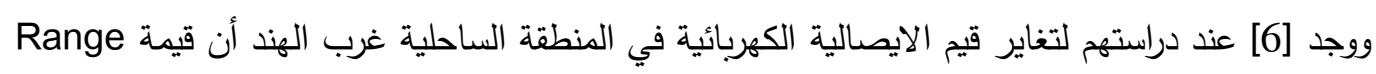

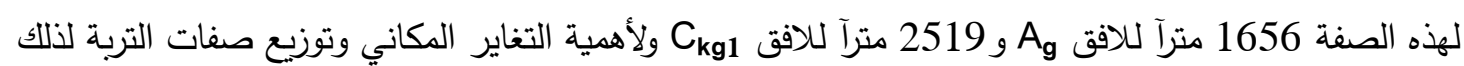

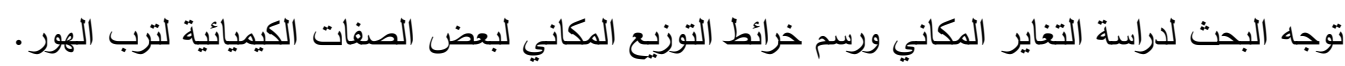
المواد وطرائق العمل

أختيرت منطقة الدراسة في محافظة ميسان هور عودة المنطقة التي تتحصر بين خطي عرض

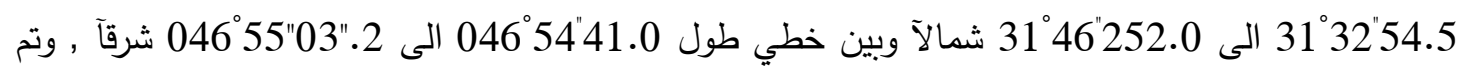

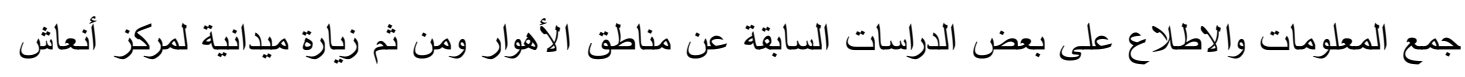

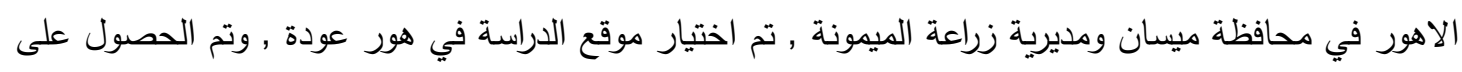

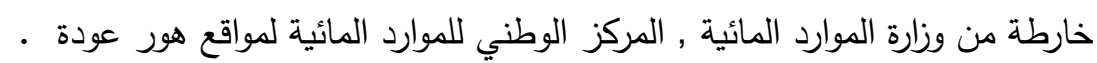

يقع هور عودة الى الغرب من نهر دجلة في محافظة ميسان على بعد 5 كم غرب قضاء الميمونة -

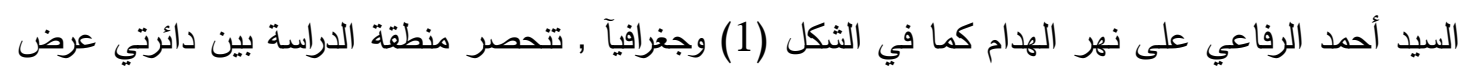

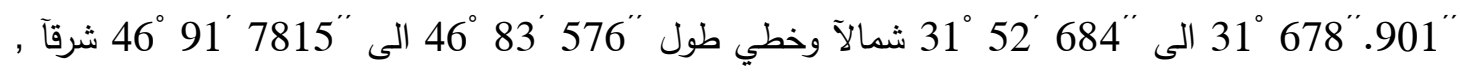
وتبلغ المساحة الكلية لهور عودة 44 الف دونم وذلك قبل عمليات التجفيف التي تعرض لها الهور مثل باقي أهوار العراق في عقد التسعينات , أما المساحة الكلية للهور بعد إعادة جزء من المياه اليه فتبلغ 12.2 ألف دونم أي أن المساحة التي تعتد مجففة فتبلغ 31.8 الف دونم [7]. 


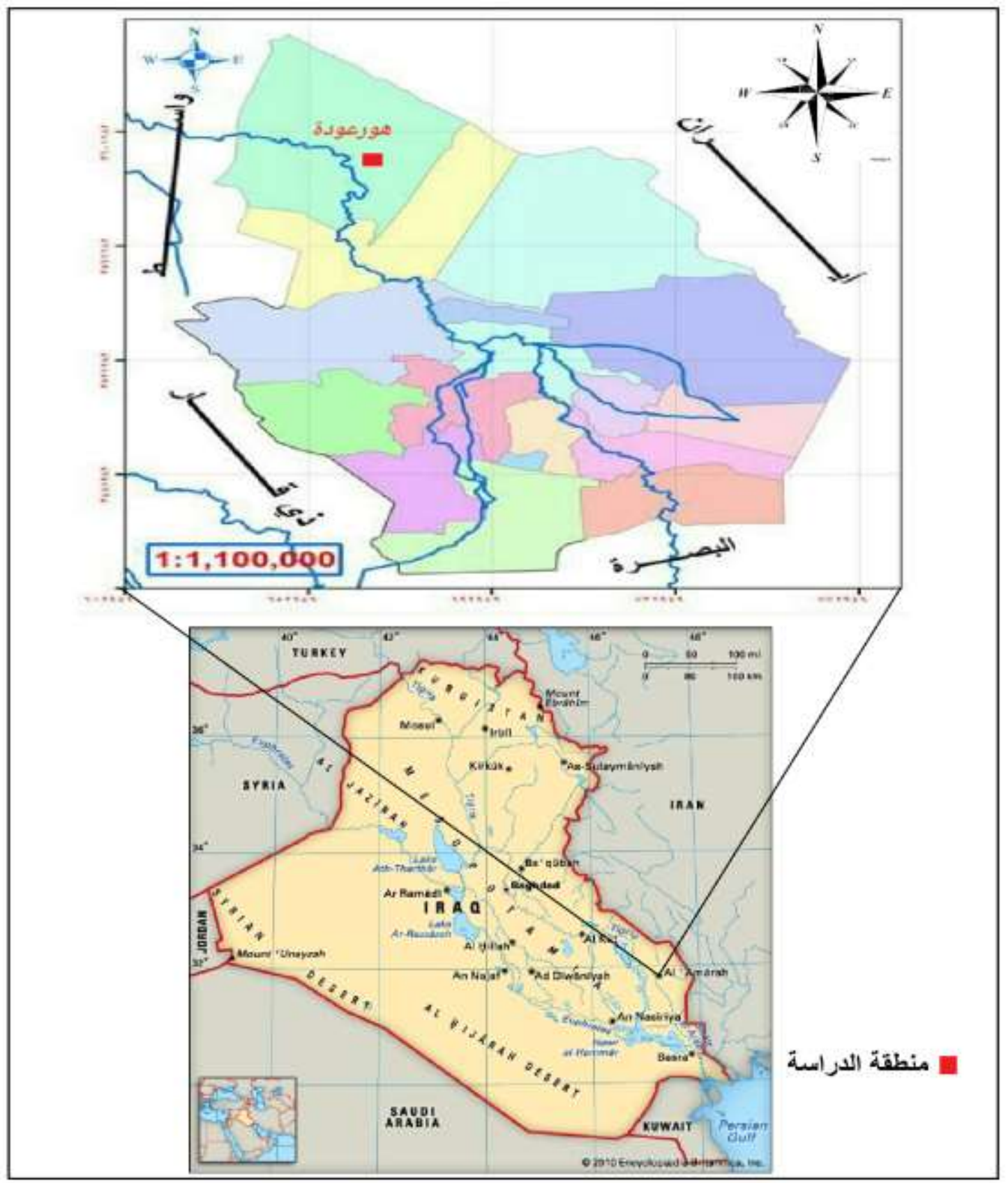

شكل 1 خارطة موقع منطقة الدراسة

اعتمادآ على المعلومات التي تم الحصول عليها في المرحلة التمهيدية , تم تحديد خط رأسي في منطقة أراضي الهور المجففة , يبدأ من الحافات القريبة من المنطقة المغورة بالمياه (هور عودة ) الى الاراضي المجفيفة

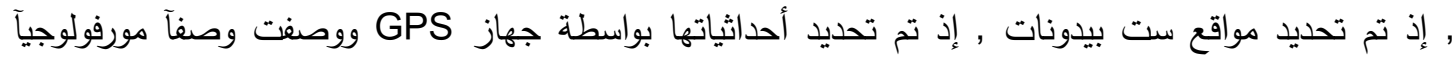
أصوليآ بحسب الاصوليات الواردة في [8]. 
بعد جلب العينات الى المختبر , تم تجفيفها هوائياً وطنها ونخلها بمنخل 2 مم بعد ذلك تم تقدير الصفات

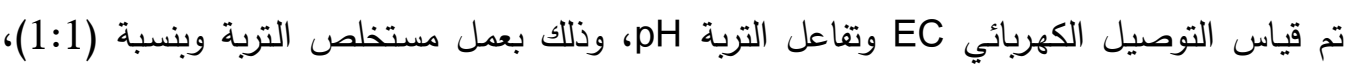
ومن ثم قياسها بواسطة الجهاز المتعدد الاغراض نوع Mi 180 Bench Meter وعمل المستخلص كما جاء

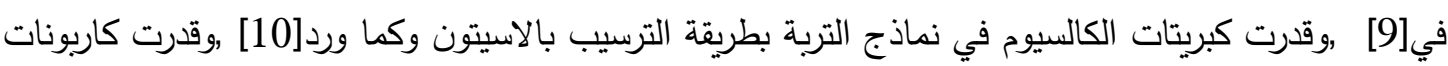

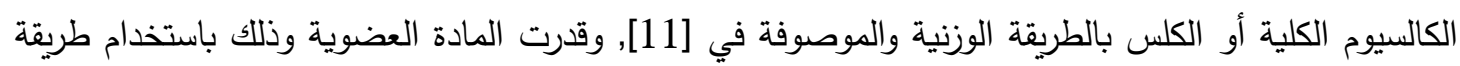
الهضم الرطب بحسب الطريقة الموصوفة في[10], وقدرت بأستعمال خلات الصوديوم وخلات الأمونيوم والموصوفة في [10] التحليل الاحصائي

تم استخدام الاحصاء الجيولوجي لغرض متابعة التغاير الككاني لبعض الصفات الرئيسية في منطقة

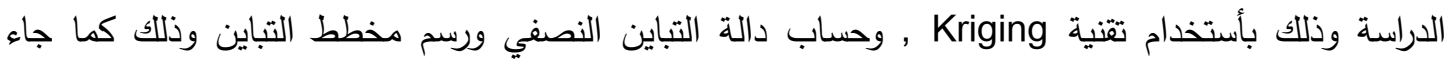

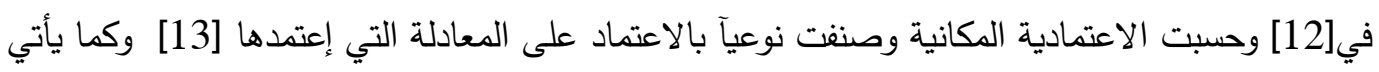
Spatiality Dependent = ( nugget / nugget + Sill )*100

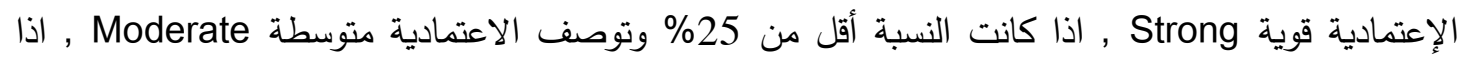

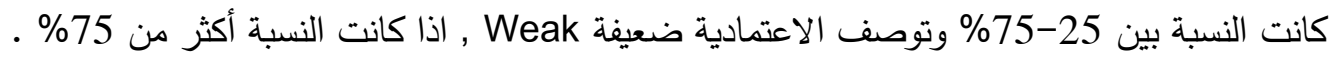

\section{النتائج والمناقشة}

التغاير الأفقي للصفات الكيميائية للنطقة الداراسة

Ec الأيصالية الكهربائية

إن صفة الملوحة يعبر عنها بالايصالية الكهربائية Ec , وعادة تكون صفة متغايرة بفعل

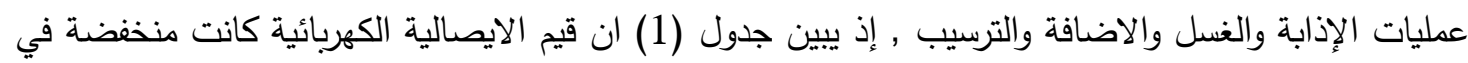

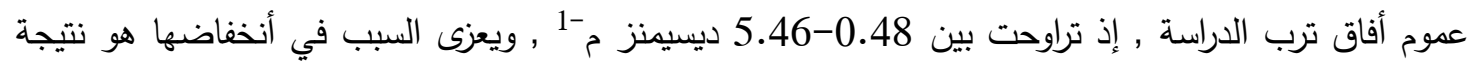
حالة التغدق وكنلك ريما ان جلب العينات كان في موسم الشتاء والتي تعمل على أذابة الترابة الاملاح ومن ثم حركتها

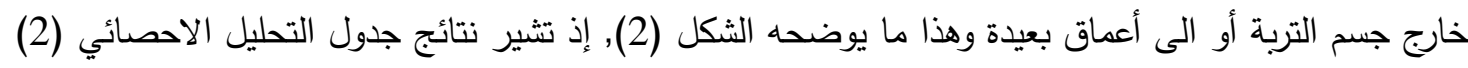
أن المسافة المؤثرة التي تصف الملوحة كانت 274 و 590 و 180 و و

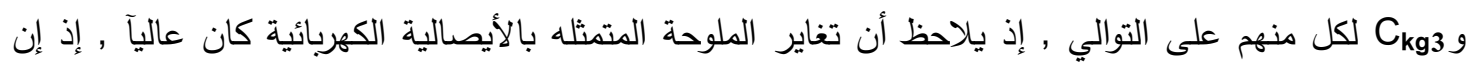

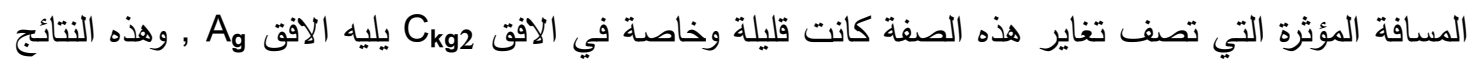

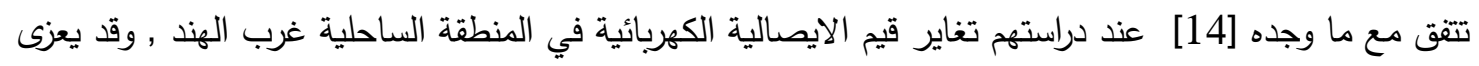

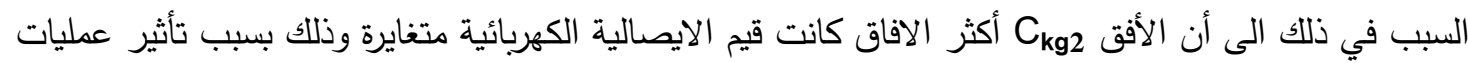

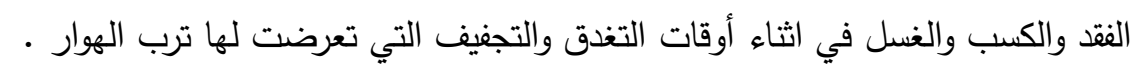

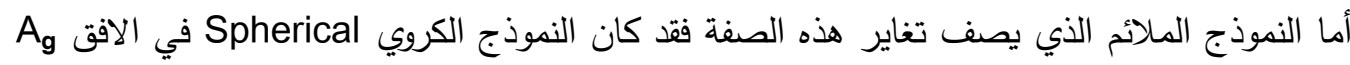

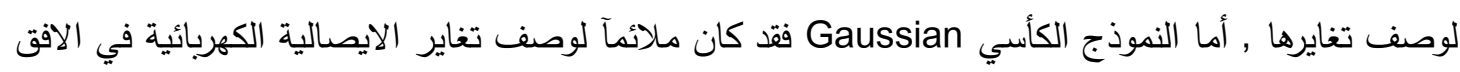

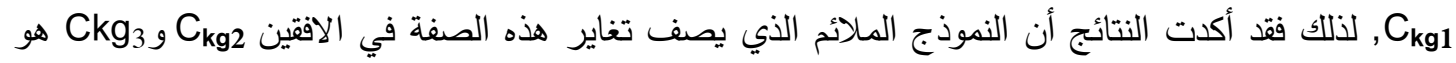

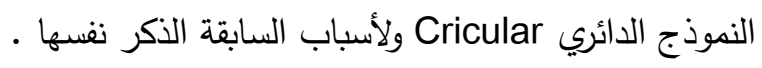


وأكد [15] أن قيمة الايصالية الكهربائية هي من أكثر الصفات تغايرآ ضمن وحدات الخارطة , إذ كانت شديدة التغاير ولاغلب أفاق وحدات الخارطة التي درسها.

جدول (1) بعض الصفات الكيميائية لترب الدراسة

\begin{tabular}{|c|c|c|c|c|c|c|c|}
\hline $\begin{array}{c}\text { الجبس } \\
\text { \% }\end{array}$ & $\begin{array}{l}\text { Caco3 } \\
\text { g. } \mathrm{Kg}^{-1}\end{array}$ & $\begin{array}{c}\text { CEC } \\
\text { Cmol. } \mathrm{Kg}^{-1}\end{array}$ & $\begin{array}{c}\text { O.M } \\
\%\end{array}$ & PH & $\begin{array}{c}\text { EC } \\
\text { ds. } m^{-1}\end{array}$ & الافاق & البيدونات \\
\hline 1.20 & 239.73 & 28.73 & 2.87 & 7.05 & 2.2 & $\mathrm{Ag}$ & \multirow[t]{4}{*}{$\mathrm{P} 1$} \\
\hline 1.31 & 297.95 & 29.25 & 1.89 & 7.31 & 1.07 & $\mathrm{Ckg}_{1}$ & \\
\hline 0.43 & 295.62 & 30.93 & 2.40 & 7.45 & 1.8 & $\mathrm{Ckg}_{2}$ & \\
\hline 0.47 & 261.51 & 33.43 & 1.12 & 7.15 & 0.94 & $\mathrm{Cg}_{3}$ & \\
\hline 0.15 & 284.28 & 37.1 & 3.01 & 7.28 & 4.1 & $\mathrm{Ag}$ & \multirow[t]{4}{*}{$\mathrm{P} 2$} \\
\hline 0.21 & 346.78 & 29.17 & 1.62 & 7.42 & 1.3 & $\mathrm{Ckg}_{1}$ & \\
\hline 0.41 & 352.47 & 38.42 & 1.79 & 7.35 & 2.6 & $\mathrm{Ckg}_{2}$ & \\
\hline 0.50 & 318.36 & 27.36 & 0.27 & 7.62 & 1.2 & $\mathrm{Cg}_{3}$ & \\
\hline 0.32 & 293.71 & 28.75 & 3.14 & 7.19 & 3.1 & $\mathrm{Ag}$ & \multirow[t]{4}{*}{ P3 } \\
\hline 0.35 & 335.41 & 26.55 & 1.31 & 7.45 & 0.88 & $\mathrm{Ckg}_{1}$ & \\
\hline 0.33 & 329.73 & 23.48 & 1.05 & 7.31 & 0.94 & $\mathrm{Ckg}_{2}$ & \\
\hline 0.36 & 324.04 & 23.65 & 0.64 & 7.40 & 0.82 & $\mathrm{Ckg}_{3}$ & \\
\hline 2.25 & 438.71 & 34.43 & 3.65 & 7.17 & 5.32 & $\mathrm{Ag}$ & \multirow[t]{4}{*}{$\mathrm{P} 4$} \\
\hline 3.14 & 375.21 & 28.66 & 1.79 & 7.29 & 1.77 & $\mathrm{Ckg}_{1}$ & \\
\hline 3.26 & 315.76 & 28.79 & 1.18 & 7.37 & 0.88 & $\mathrm{Ckg}_{2}$ & \\
\hline 4.61 & 379.94 & 25.03 & 0.77 & 7.43 & 1.63 & $\mathrm{Ckg}_{3}$ & \\
\hline 0.82 & 306.53 & 25.05 & 4.16 & 7.19 & 5.46 & $\mathrm{Ag}$ & \multirow[t]{3}{*}{ P5 } \\
\hline 0.87 & 369.53 & 24.20 & 2.10 & 7.59 & 1.36 & $\mathrm{Ckg}_{1}$ & \\
\hline 0.73 & 301.31 & 22.32 & 1.42 & 7.42 & 1.24 & $\mathrm{Ckg}_{2}$ & \\
\hline 0.89 & 324.05 & 22.38 & 0.88 & 7.22 & 1.25 & C3 & \\
\hline 2.20 & 308.90 & 30.34 & 4.50 & 7.21 & 2.61 & $\mathrm{AP}$ & P6 \\
\hline 0.25 & 358.16 & 26.97 & 0.70 & 7.39 & 1.01 & $\mathrm{Ckg}_{1}$ & \\
\hline 0.31 & 312.68 & 22.08 & 0.51 & 7.42 & 0.48 & $\mathrm{Ckg}_{2}$ & \\
\hline 0.37 & 332.08 & 18.66 & 1.37 & 7.38 & 0.93 & $\mathrm{Ckg}_{3}$ & \\
\hline 0.99 & 325.10 & 25.70 & 1.80 & 7.34 & 1.87 & & المتوسط العام \\
\hline
\end{tabular}



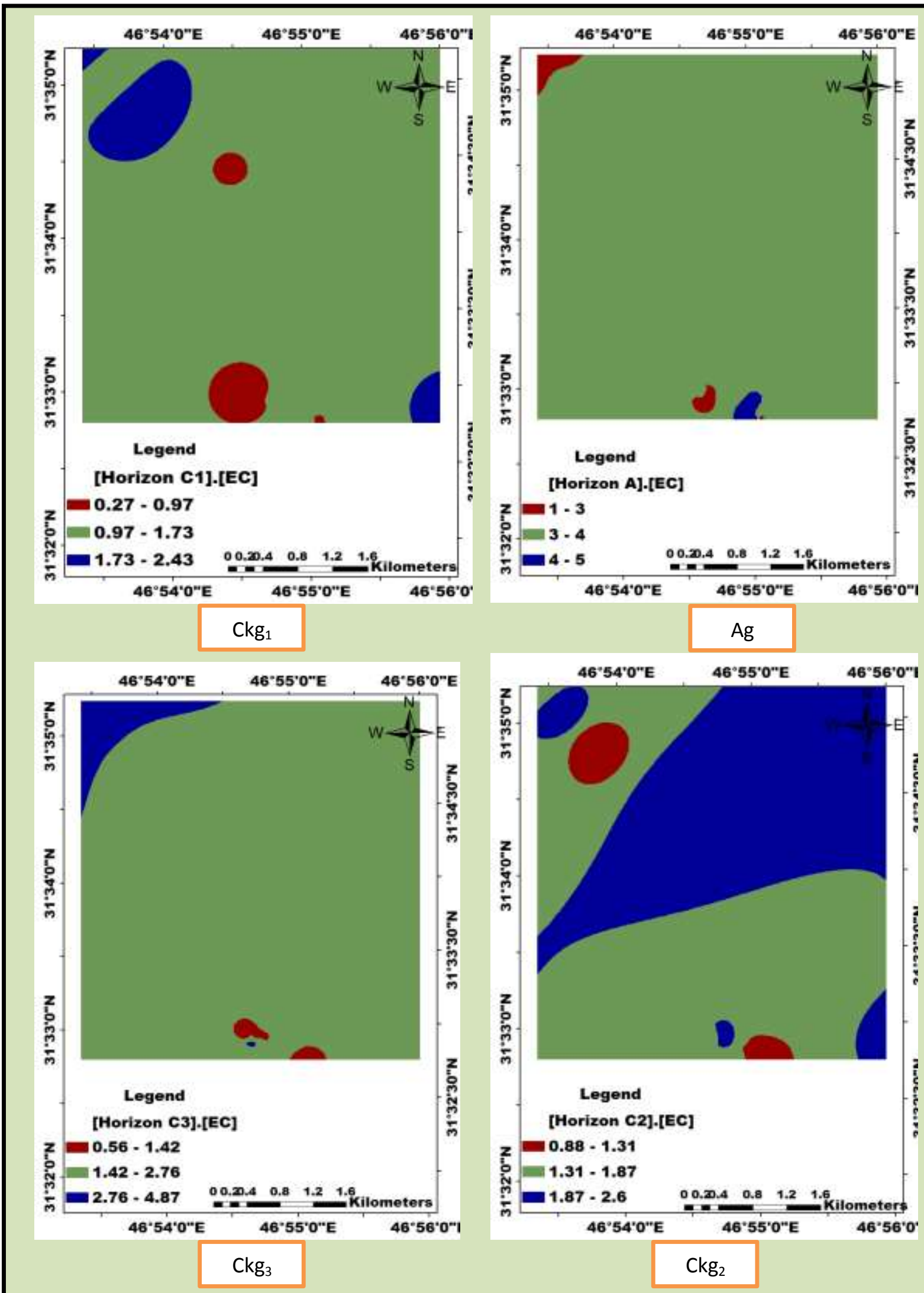

شكل 2 خارطة التوزيع المكاني لل ECC لافاق ترب الدراسة 
وهذه النتائج تتفق مع ما وجده [19] عند دراستهم تغاير تفاعل ترب كالفورنيا , إذ حصلوا على Range للافق السطحي 1650 مترآ , وللافق تحت السطحي 2600 مترآ , وعزوا السبب الى أن الترب الكلسية تمتاز بالخاصية الثعرية.

جدول (2) التحليل الاحصائي للصفات الكيميائية لترب الدراسة بأستخدام الأحصاء الجيولوجي

\begin{tabular}{|c|c|c|c|c|c|c|c|}
\hline Model & الاعتمادية المكانية & Sill & Partial Sill & Nugget & $\begin{array}{c}\text { Range } \\
\text { (m) }\end{array}$ & الصفة & الافاق \\
\hline Sperical & Strong & 2.499 & 2.499 & 0 & 274 & $\mathrm{Ec}$ & \multirow{6}{*}{$\mathrm{Ag}$} \\
\hline Circular & Strong & 0.041 & 0.04 & 0.001 & 3382 & $\mathrm{pH}$ & \\
\hline Circular & Strong & 0.64 & 0.63 & 0.01 & 591 & OM & \\
\hline Circular & Strong & 22.956 & 22.416 & 0.54 & 401 & CEC & \\
\hline Sperical & Strong & 1995.4 & 1995.3 & 0.1 & 4671 & $\mathrm{CaCo} 3$ & \\
\hline Sperical & Strong & 0.67 & 0.67 & 0 & 240 & Gypsum & \\
\hline Gaussian & Strong & 0.44 & 0.43 & 0.01 & 590 & Ec & \multirow{6}{*}{$\mathrm{C} 1$} \\
\hline Sperical & Strong & 0.04 & 0.04 & 0 & 2245 & $\mathrm{pH}$ & \\
\hline Sperical & Strong & 0.22 & 0.22 & 0 & 160 & OM & \\
\hline Sperical & Strong & 4.34 & 4.22 & 0.12 & 784 & CEC & \\
\hline Sperical & Strong & 9178.24 & 9178.2 & 0.04 & 1121 & $\mathrm{CaCo} 3$ & \\
\hline Sperical & Strong & 1.11 & 1.11 & 0 & 109 & Gypsum & \\
\hline Circular & Strong & 0.4 & 0.38 & 0.02 & 180 & Ec & \multirow{6}{*}{$\mathrm{C} 2$} \\
\hline Circular & Strong & 0.044 & 0.044 & 0 & 851 & $\mathrm{pH}$ & \\
\hline Sperical & Strong & 0.21 & 0.2 & 0.01 & 1300 & OM & \\
\hline Circular & Strong & 31.61 & 30.58 & 1.03 & 787 & CEC & \\
\hline Circular & Strong & 397.77 & 397.77 & 0 & 1851 & $\mathrm{CaCo} 3$ & \\
\hline Sperical & Strong & 0.93 & 0.92 & 0.01 & 420 & Gypsum & \\
\hline Circular & Strong & 2.1386 & 2.1386 & 0 & 484 & Ec & \multirow{6}{*}{$\mathrm{C} 3$} \\
\hline Circular & Strong & 0.071 & 0.07 & 0.001 & 1696 & $\mathrm{pH}$ & \\
\hline Circular & Strong & 0.125 & 0.125 & 0 & 368 & OM & \\
\hline Circular & Strong & 45.468 & 45.468 & 0 & 245 & CEC & \\
\hline Circular & Strong & 5000 & 5000 & 0 & 1967 & $\mathrm{CaCo} 3$ & \\
\hline Circular & Strong & 3.1143 & 3.1143 & 0 & 330 & Gypsum & \\
\hline
\end{tabular}


Journal of University of Babylon, Pure and Applied Sciences, Vol.(27), No.(1): 2019

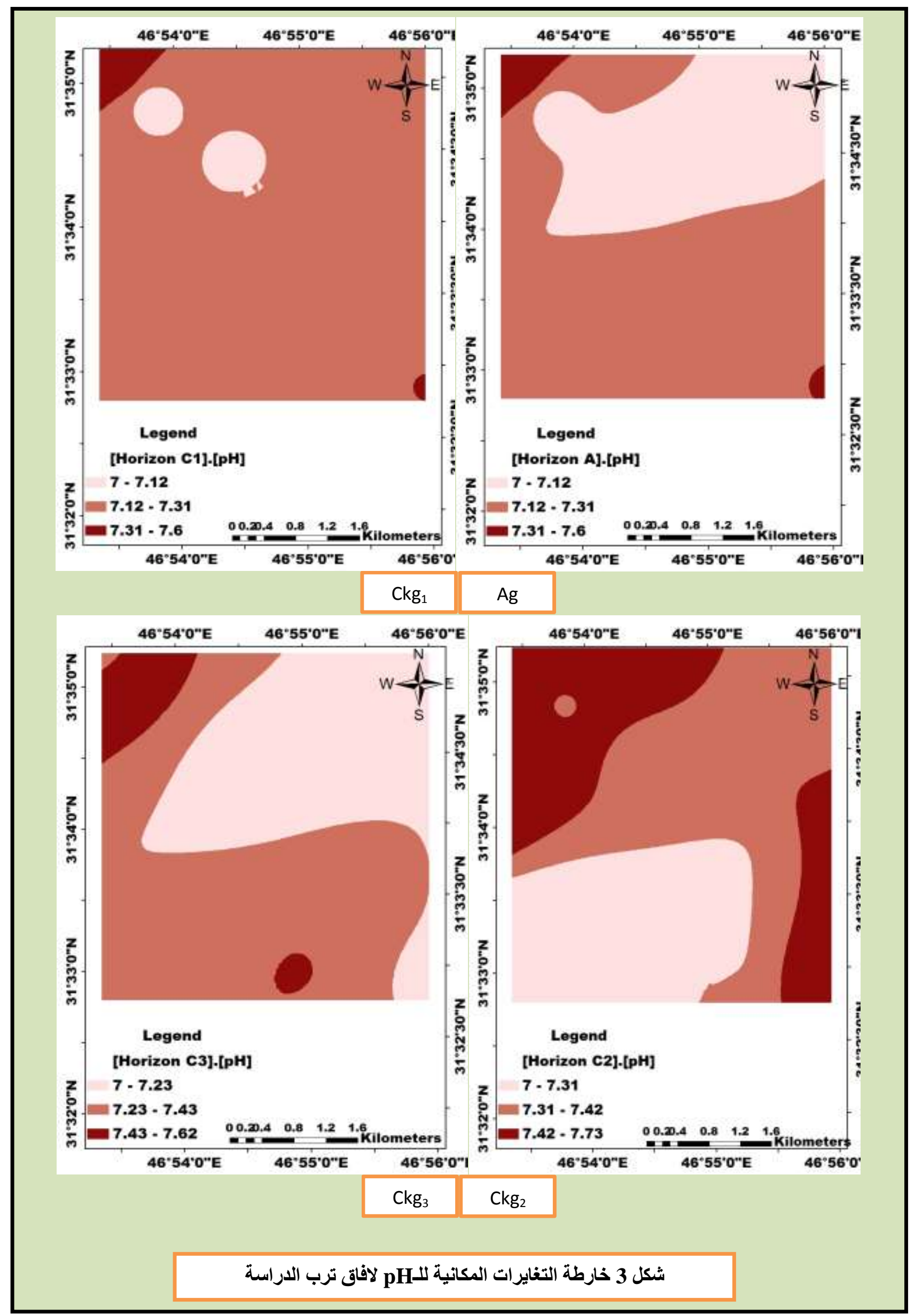


إن محتوى ترب الدراسة من المادة العضوية والموضح في جدول (1) تشير الى حالة من التباين في محتوى وتوزيع المادة العضوية في آفاق بيدونات الدراسة , ويلاحظ أرتفاعها في آفاق الترب وخاصة الإفاق

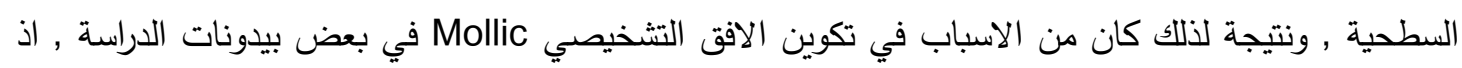

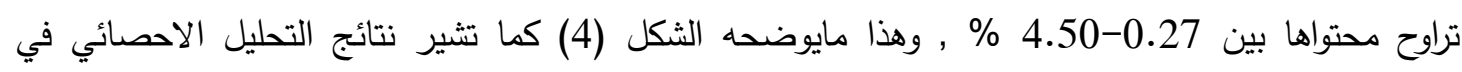

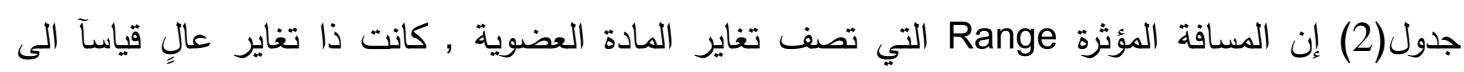
المسافة الكلية , إذ بلغت 591 و 160 و 1300 و368 مترآ للافاق Ag و

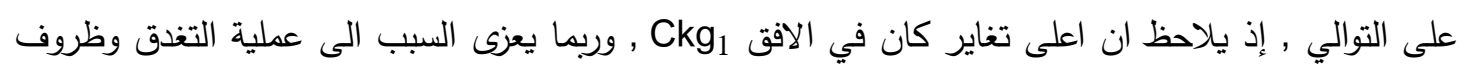

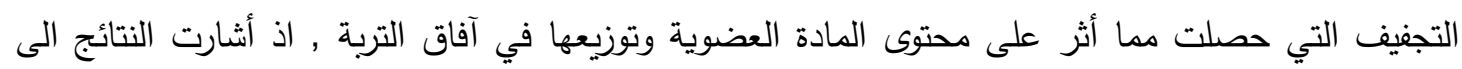
تغاير عالٍ في هذه الصفة , وربما يعود السبب في تغايرها الى ظروف التغدق والاختزال التي تعمل على تقليل

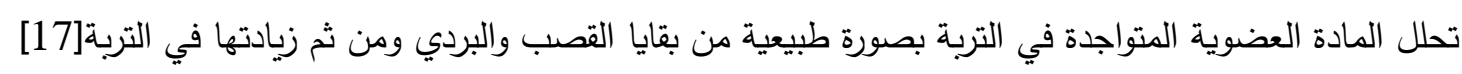

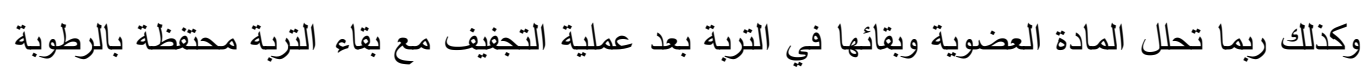
ولاسيَما ان النسجات السائدة في تلك التربة كانت النسجات الناعمة وسيادة مفصول الطين.

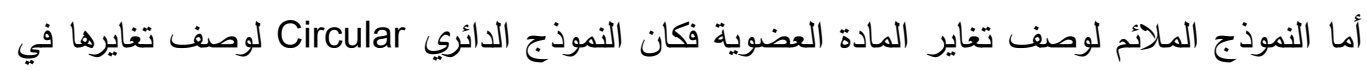

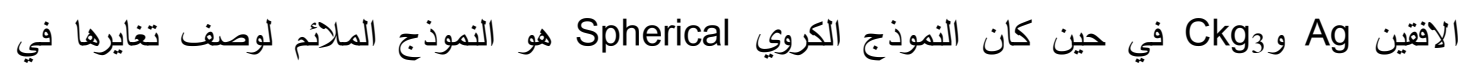
الافقين 


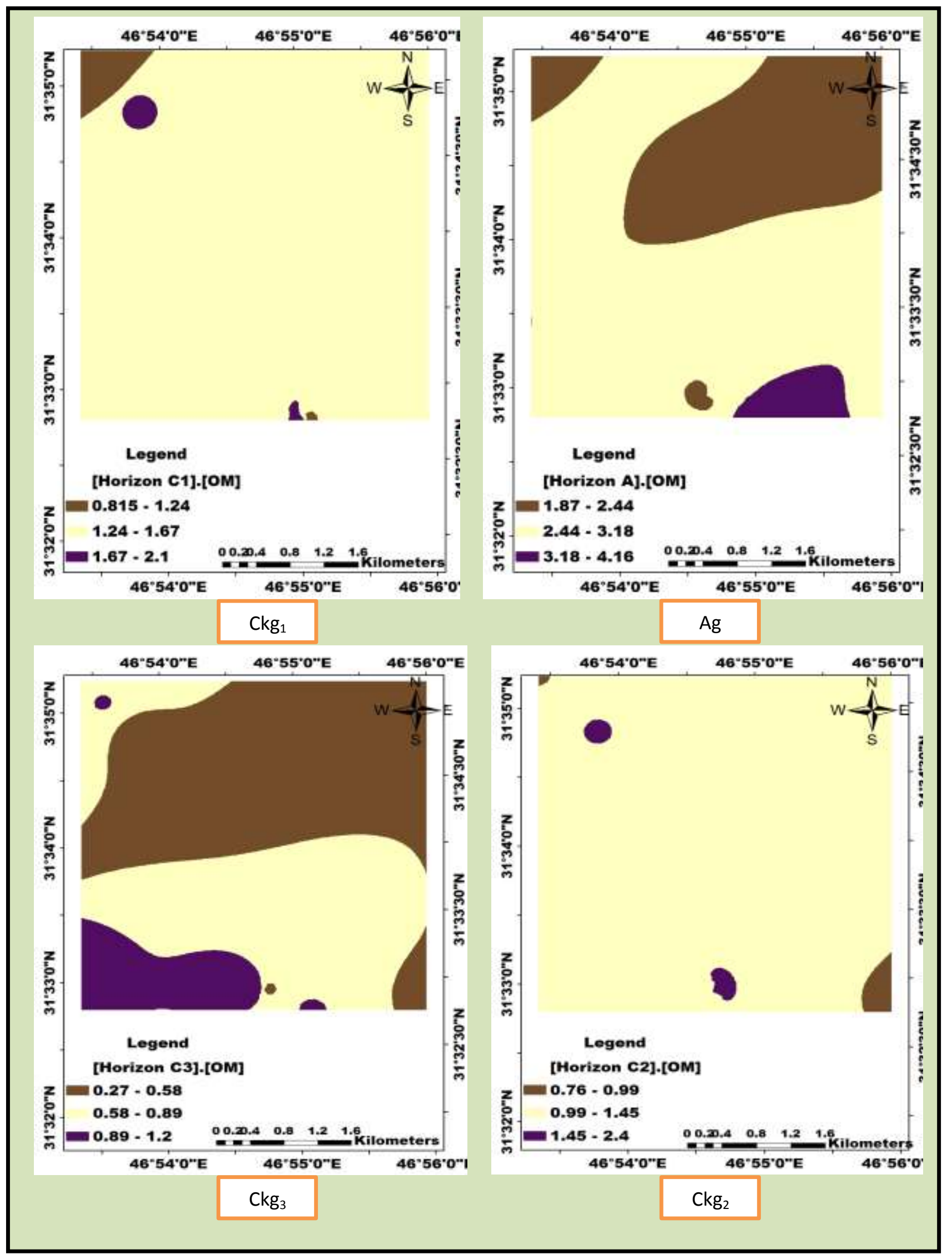

شكل 4 خارطة التغايرات المكانية للO.M لافاق ترب الاراسة 
Journal of University of Babylon, Pure and Applied Sciences, Vol.(27), No.(1): 2019

الطين وتفاعل التربة وهذا يتفق مع ما وجده [21] إذ وجدوا ان المسافة المؤثرة للسعة التبادلية الايونية

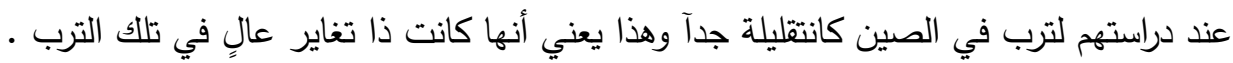

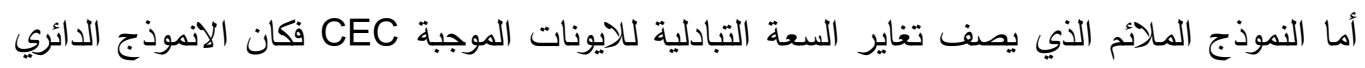
فكان النموذج الملائم لوصف تغاير هذه الصفة فيه كان Circular النموذج الكروي Spherical , وهذه النتائج تتوافق مع نتائج النماذج التي تصف تغاير الطين والمادة العضوية [22] مما يؤكد تأثير وارتباط هذه الصفة بتغايرها وهذا ما أكده) , 
Journal of University of Babylon, Pure and Applied Sciences, Vol.(27), No.(1): 2019

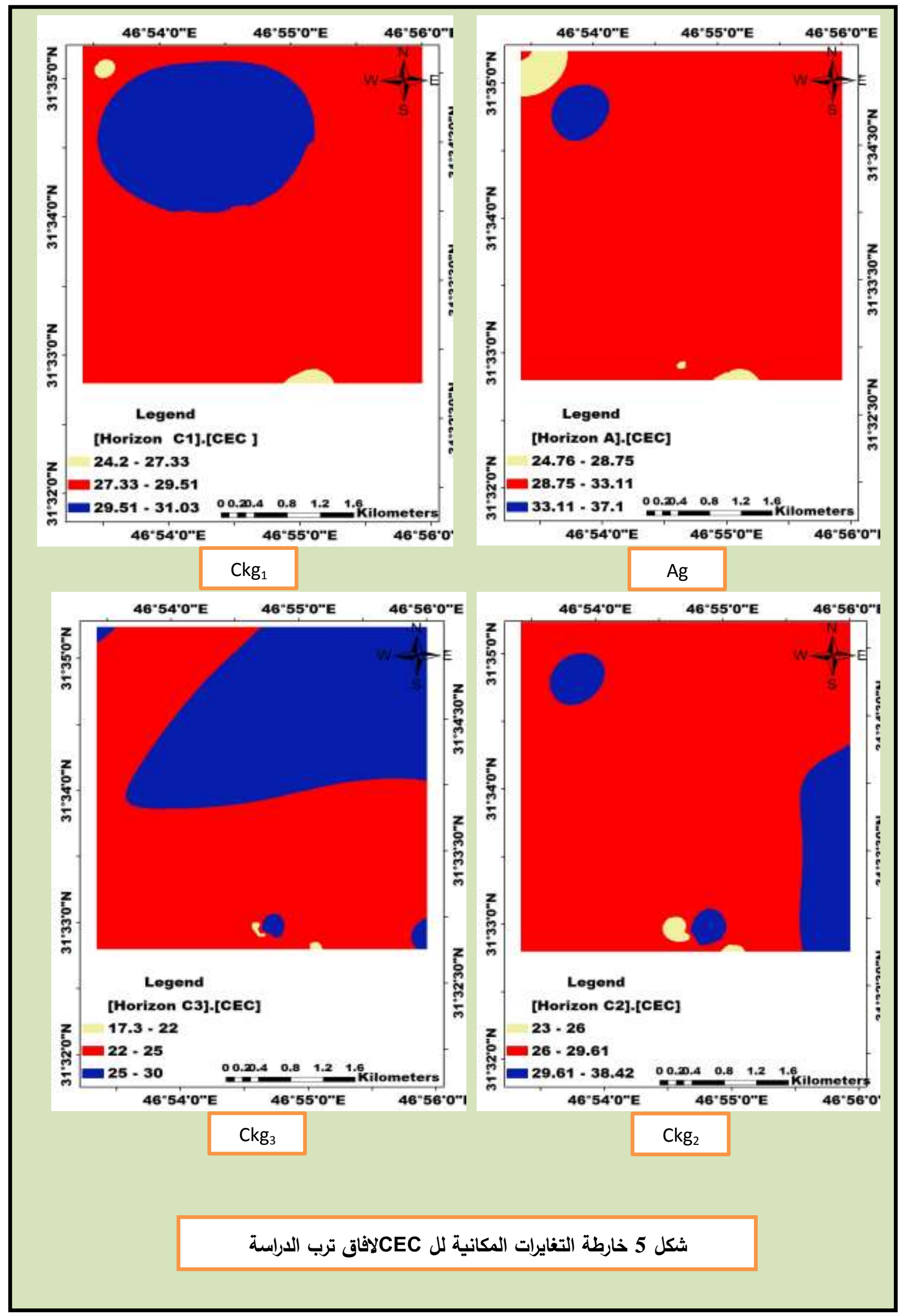


الافق Ag للبيدون 4 كما موضح في شكل (6), ويعزى السبب في ذللك الى حالة التغدق التي سببت في إعادة

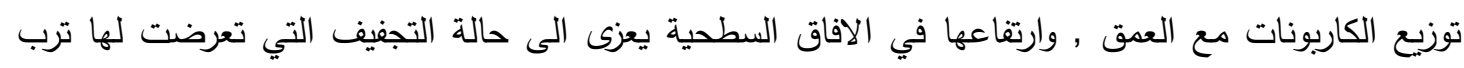

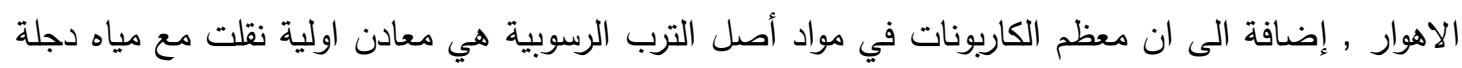
والفرات وترسبت بشكل دقائق ناعمة [23] ويلاحظ وجود تجانس في توزيعها في بيدونات الدراسة بسبب كونها مشتقة من مادة أصل واحدة وظروف ترسيب مشابهة .

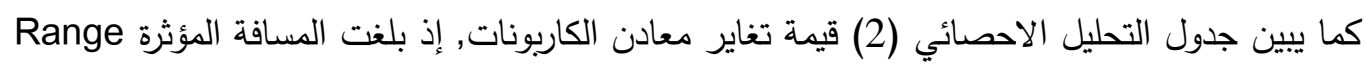

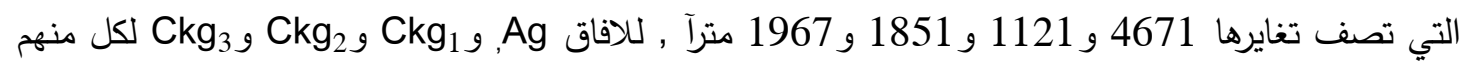

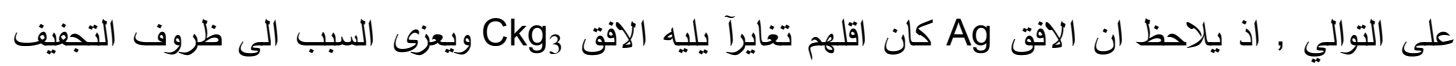

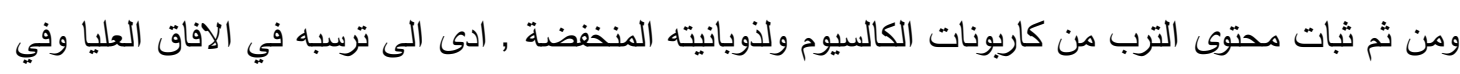

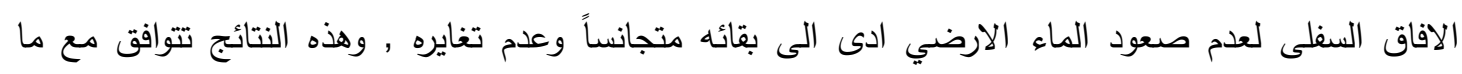

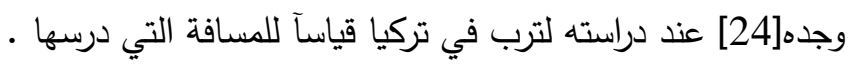




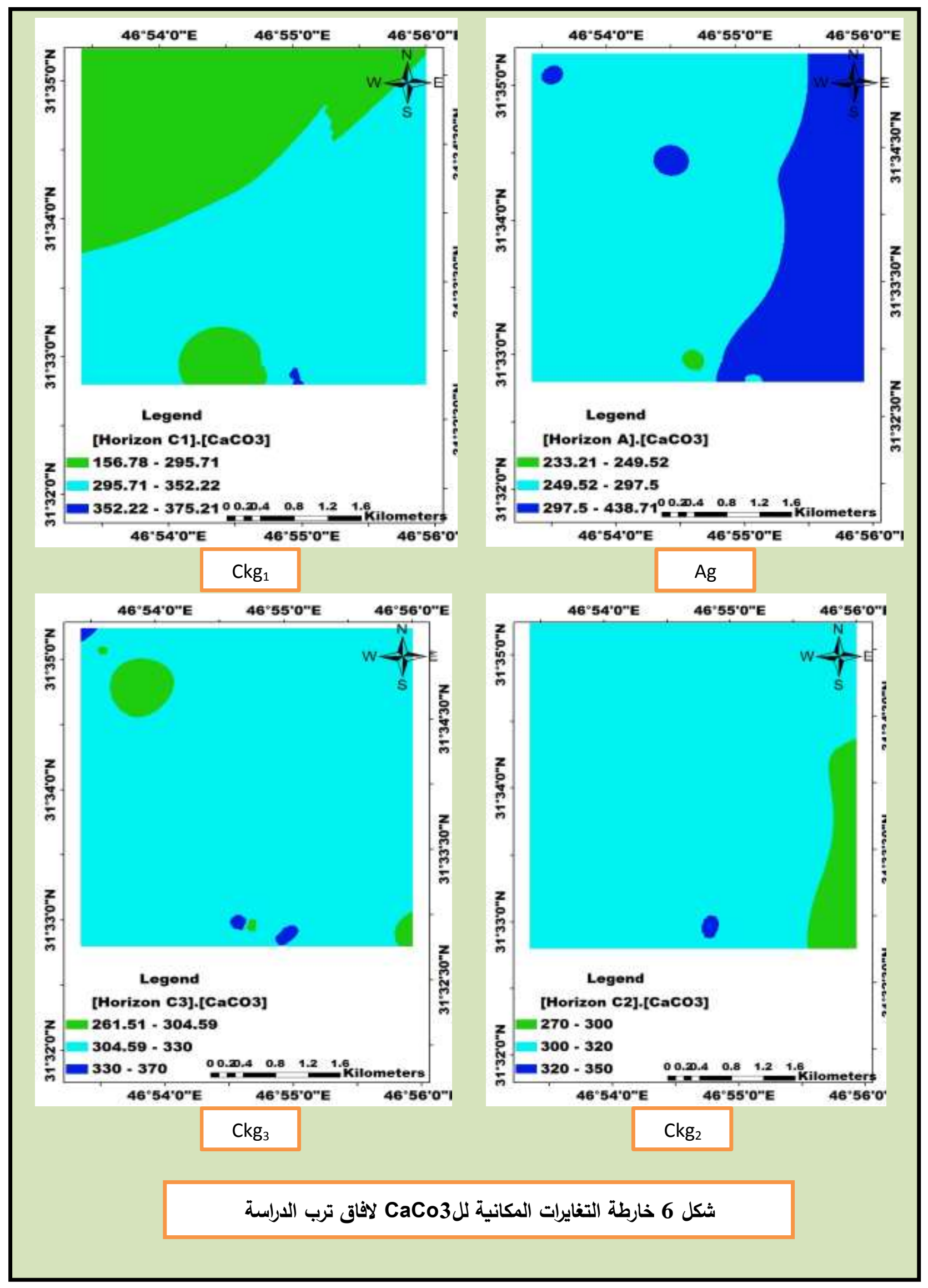


أما النموذج الملائم الذي يصف تغاير الجبس فكان الانموذج الكروي Spherical لجميع الافاق , ما

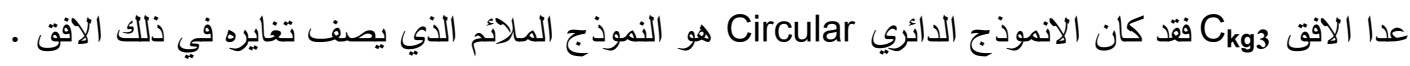
تم تصنيف ترب منطقة هور عودة بموجب التصنيف الحديث للترب [27] وطبقآ لهذا النظام تقع ترب

$$
\text { هور عودة في رتبتين هما : }
$$

Entisols-1 من ترسبات الانهار وهي تضم المجموعة العظمى Great group المسماة aquifluvents لأنها ترب رطبة كانت متغدقة لهذا فأن النظام الرطوبي لها هو من نوع aquic إذ تكون التربة مشبعة بالماء جزئياً أو كليآ لفترة

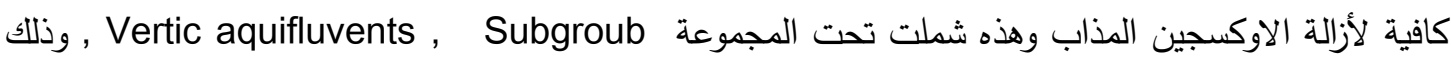
لوجود التشققات في ترب تلك المنطقة , وربما لإحتوائها على نسبة طين عالية ولعمق أكثر من 50 سم وربما لوجود معادن السمكتايت وقد شملت البيدونات 1 و 2 بالرتبة الحديثة التكوين .

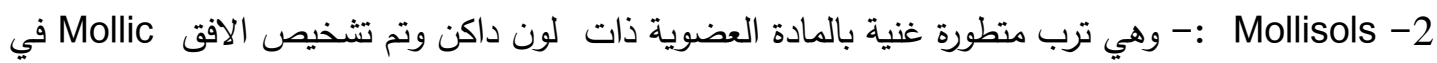
تلأك الترب وتضم تحت الرتبة aquolls Suborder وذللك لانها ترب كانت متغدقة لمدة من الزمن والمجموعة

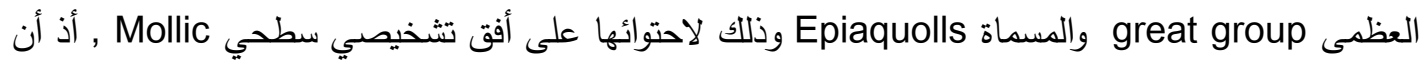
تعني باليونانية فوق ذات نظام رطوبي aquic وذللك لوجود أفق تجمع الطين بصورة بدائية المسماة

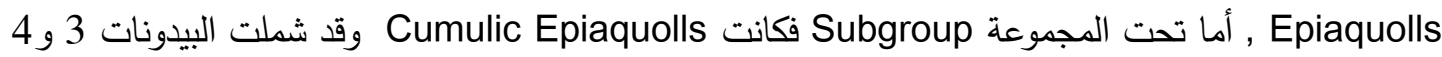

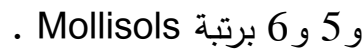


Journal of University of Babylon, Pure and Applied Sciences, Vol.(27), No.(1): 2019

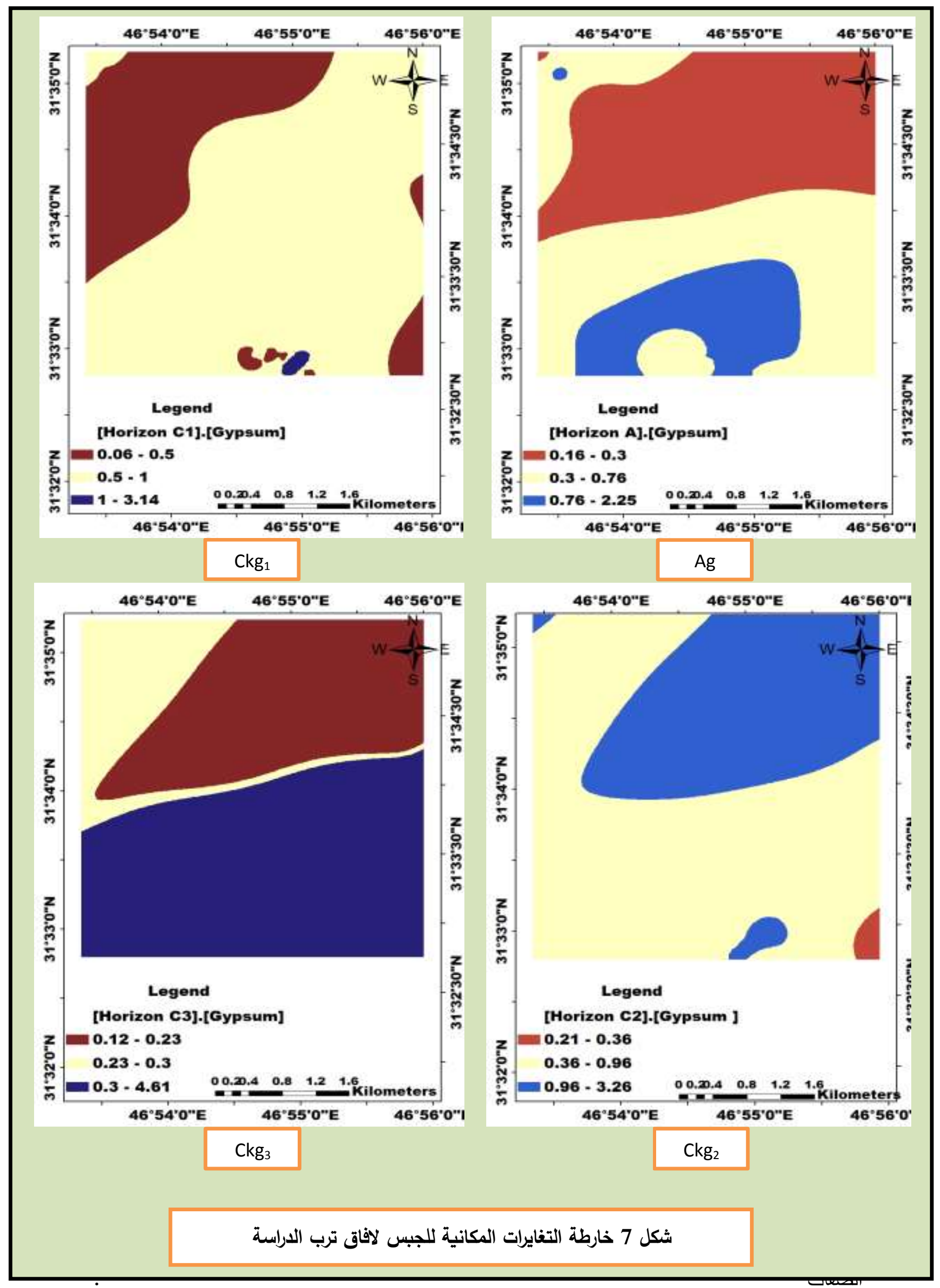




\section{CONFLICT OF INTERESTS.}

There are non-conflicts of interest.

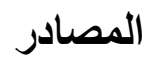

[1] Ozgoz , E., F. Akbas , M.Cetin ,S. Ersatin and H. Gunal ( 2007) . Spatial Varrability of soil physical properties as affected by different tillage system . New Zealand Journal of Crop and Horticultural Science . 35: 1-13.

[2] Lark , R.M. ( 2002 ) . Optimized spatial sampling of soil for estimation of the Variogram by maximum likelihood . Geoderma 105.2002.49-80 .

[3] AlMaaofi ,Sama ,Ali Doubul ,Hamid Al-Saad ( 2012) .Mesopotamian Mard land . salinization problem .Journal of Environmental protection,v.3.

[4] Mulla , D.J. and A.B Mc Bratney ( 2000 ). Spatial variability of soil acidity attributes and the spatialization of liming requirement for corn . Ciencia Agrotecnologia .33(5) 321-352 New Jersey ,390pp-second Edition .

[5] Aishah , A. W. , S. Zauyah , A. R. Anvar ,C.I. Fauziah ( 2010 ) . spatial variability of selected chemical characteristics of padoly soils in sawah sempadan, Selangor, Malaysia . Malaysia . J of soil Sci .vol 14: 27-39 .

[6] Behera , S. K. , K. Suresh , B. N. Raol , R. K. Mathur , A. K. Shukla , K.(2016) Manorama , K. Ramachandrudu , P. Harinarayar C. Properties Varies in oil palm ( Elaeis guineensis Jacq ) Plantations of west Coastal area of India . Solid Earth (7) 979 -993 .

$$
\text { [7] وزارة الموارد المائية ، مركز انعاش الاهوار، بيانات غير منشورة ، } 2017 \text {. }
$$

[8] Soil Survey division Staff ( 1993 ) .Soil Survey manovl , USDD . hand book No 18 us Gorement Printing office Waching .tor . Dc. 20402 .

[9] Page, A. L., Miller, H., and D. R. Keeny. (1982). Method of soil analysis. Part (2) chemical and biological properties, Am. Soc. Agron. Inc., Pub. Madison, Wissonsinsin. USA.

[10] Jackson, M. L. (1958). Soil chemical analysis. Prinite-Hall, Inc. Englewood. Cliffis, N.J.

[11] Hesse ,P. R. ( 1971 ) .Atextbook of soil chemical analysis .William Clowes and Sops limited London, pp: 461-476 .

[12] Krasilinkov , P. ; F. Carre ; and L. Montanarella ( 2008 ). Soil geography and geostatistics, J. Am, Vol. 5 ( 4 ).

[13] Iqbal , J. , J. A. Thomasson , N. Johien ., R.O. Phillip. , and D. W. Frank D. 2005 ). Spatial variability analysis of soil Physical .

[14] Behera, S. k., k. Suresh , B. N. Raol ,R. K. Mathur , A. K. Shukla , K. Manorama , K . Ramachandrudu , P. Harinarayana , C. Prakash . ( 2016 ). spatial variability of some soil properties varies in oil palm (Elaeis guineensis Jacq.) plantations of west coastal area of India . Solid Earth .(7) 979-993 .

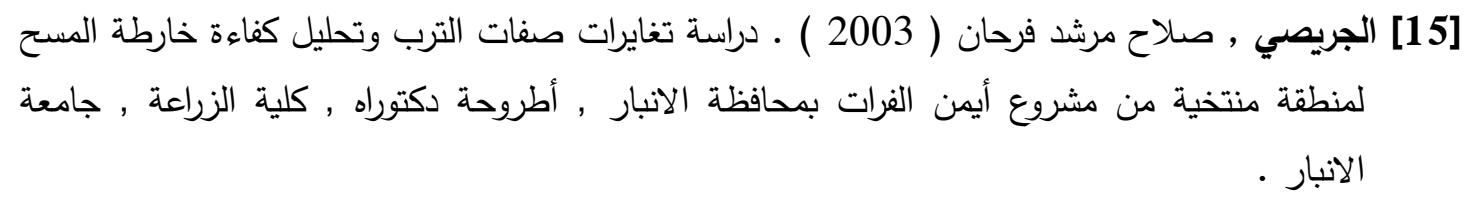


[16] Delever, P. ,( 1962 ) . Properties of Saline Soil in Iraq . Neth . J. Agr. Sci. , 10 : $194-210$.

[17] Meresht, Javed Seyedmohammadi (2013). The effect of toposequence on physical and and chemical characteristics of paddy Soils of Guilan province, Northern Iran, Rasht, African Journal of Agricultural .

[18] عبد الله, حازم محمود أحمد (2006). ثرموديناميكية وحركية ذوبان كاربونات الكالسيوم في بعض الترب الكلسية / العراق , أطروحة دكتوراه ـ قسم علوم التربة والمياه ـ كلية الزراعة. جامعة الموصل.

[19] Corwin, D.L., Lesch, S.M. (2005). Apparent Soil electrical conductivity measurements in agriculture . Computers and Electronics in Agricultvre. 4611 -43 .

[20] الجبوري, صبار راهي جاسم (2012). أصل ووراثة معدن الجبس في بعض الترب الجبسية من العراق. أطروحة دكتوراه ـ كلية الزراعة - جامعة بغداد.

[21] Young - dong; F. Wang ; N.L. Xvan ; (2008) . Spatial Variability of Soil Cation exchange Capacity in Hilly Tea Plantation Soils under different Sampling Scales . Agr . Sci . in China, 7 (1) .

[22] Chaikaew, Pasicha and Suchana Chavanich (2017). Spatial Voriability and Relationship of Mangrove Soil Organic Matter to Organic Carbon . Applied and Environmental Soil Science Vol . 2017 , Article ID 4010381, P. 9.

[23] Buringh , P.(1960) . Soil and Soil Condition in Iraq .Ministry of Agri Baghdad, Iraq .

[24] Akbas , F. (2014) . Spatial Variability of soil Color Parameters and soil Properties in an alluvial soil . Academic Journals . vol . 9 (12), pp. 1025 - 1035.

[25] حن , خالد فالح (1981). دراسة معادن وبعض صفات ترب منطقة الجزيرة تلعفر -بعاج. رسالة

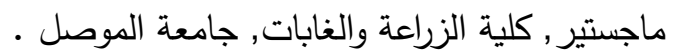

[26] Denton , O.A., V.O. Aduromigba - Modupe , A.O. Ojo , O.D. Adeoyolanu , K. S. Are, A.O. Adelana , A.O. Oyedele , A.O. Adetayo and A.O. Oke. (2017). Assessment of spatial variability and mapping of soil properties for svstainable agricultural production using geographic in for mation system techniques (GIS) . Cogenl Food \& Agriculture, $3: 127$.

[27] Soil Survey Staff (2010) . Keys to soil Taxonomy. 12 . Unated States. Department of Agriculture natural resources conservation service $\mathrm{Sw}$. Washington, D C . 